\title{
CAMBIO Y CONTINUIDAD EN EL PENSAMIENTO ESTRATÉGICO ESTADOUNIDENSE DESDE EL FINAL DE LA GUERRA FRÍA*
}

\author{
Change and Continuity in the United States' Strategic \\ Thought since the End of the Cold War
}

\section{GUILLEM COLOM PIELLA}

Universidad Pablo de Olavide / Universidad Pontificia de Comillas

\begin{abstract}
RESUMEN
El artículo estudia la evolución del pensamiento estratégico estadounidense desde el final de la Guerra Fría hasta la actualidad. Se argumenta que su desarrollo se ha producido en tres etapas -la inmediata posguerra fría (1989-2001), la guerra contra el terror (2001-12) y la actualidad (2012-)-, cada una de ellas condicionada por un paradigma que ha restringido el pensamiento estratégico, la política de defensa y la actuación militar del país. Sin embargo, a pesar de las transformaciones experimentadas para anticiparse a los riesgos o responder a las amenazas, este ha mantenido una gran continuidad.
\end{abstract}

Palabras clave: Pensamiento estratégico, política de defensa, posguerra fría, guerra contra el terror, Estados Unidos.

\begin{abstract}
The article is aimed at studying the evolution of the United States' strategic thought since the end of the Cold War until the present day. Its development can be divided into three different periods - the immediate post-Cold War (1989-2001), the war on terror (2001-12) and nowadays (2012-) - any of which based on a paradigm that has determined the country's strategic thought, defence policy and military posture. Regardless of those transformations aimed at anticipating to the risks or respond to the threats, the American strategic thought has maintained a great deal of continuity.
\end{abstract}

Key words: Strategic Thought, Defence Policy, Post-Cold War, War on Terror, United States.

* Agradezco los comentarios que los evaluadores anónimos realizaron a la versión preliminar del texto. Sus propuestas han sido de gran ayuda para elaborar el trabajo final. 


\section{INTRODUCCIÓN}

Desde el final de la Guerra Fría han sido muchos los expertos que han tratado de identificar y estudiar las dinámicas del mundo actual. El presente artículo centrará su interés en el estudio de un aspecto muy concreto pero muy relevante para el estudio de las Relaciones Internacionales, como es la evolución que ha experimentado el pensamiento estratégico y su influencia en la configuración de la política de seguridad estadounidense desde la caída del Telón de Acero hasta la actualidad.

Para lograr este objetivo, el trabajo se fundamentará en dos hipótesis. En primer lugar, se argumenta que pueden distinguirse tres etapas en la evolución del pensamiento estratégico estadounidense desde el fin de la Guerra Fría':

- Una primera etapa (1989-2001) que, comprendida entre la caída del Muro de Berlín y los ataques sobre Nueva York y Washington, se caracterizó por una aparente estabilidad global y la hegemonía americana. Durante esta pausa estratégica ${ }^{2}$ que motivó el cobro del dividendo de la paz y la reestructuración de la arquitectura de seguridad y defensa estadounidense, la Revolución en los Asuntos Militares fue el paradigma dominante en su análisis estratégico.

- Una segunda etapa (2001-2011) comprendida entre los sucesos del 11-S hasta la muerte de Osama Bin Laden y concretada en la guerra contra el terror. ${ }^{3}$ Las nuevas amenazas a la seguridad internacional, las nuevas misiones de las fuerzas armadas y los problemas surgidos durante las campañas afgana e iraquí motivaron que la Transformación como imperativo estratégico se consolidara como el foco del análisis militar estadounidense.

- Una tercera etapa (2011-) iniciada tras la eliminación de Bin Laden y caracterizada por la consolidación de nuevas potencias regionales, una permanente inestabilidad global y el declive de la hegemonía norteamericana. Esta transición estratégica ${ }^{4}$

1 También podría argumentarse que cada uno de estos tres periodos se ha caracterizado por un paradigma de política exterior, ya que en la inmediata posguerra fría el país adoptó una acción multilateral primacista que se transformó en unilateralismo militante tras los sucesos de 2001 y que ha acabado desembocando en un multilateralismo con tendencias aislacionistas. No obstante, su estudio se dejará para posteriores trabajos que complementen el presente artículo.

2 El concepto de Strategic Pause -entendida esta como el periodo comprendido entre la desaparición de la Unión Soviética y la emergencia de un nuevo competidor global- fue empleado por primera vez de forma oficial por el Secretario de Defensa Les Aspin en la Bottom-Up Review de 1993. Ampliamente utilizada por la Administración Clinton para justificar sus decisiones en materia de defensa, fue muy criticada por la oposición republicana, que pretendía aprovechar la aparente estabilidad que brindaba la posguerra fría para transformar la maquinaria militar estadounidense para enfrentarse a los retos futuros.

3 Aunque el concepto de Global War on Terror fue definido por el presidente George W. Bush tras el 11-S, formalizado en la National Security Strategy de 2002 y desde entonces ha sido profusamente empleado para definir las actividades que Washington y países afines han llevado a cabo para combatir a la Yihad global; con el nombramiento de Barack H. Obama la guerra contra el terror ha sido eliminada de la terminología política estadounidense y sustituida por los conceptos de Overseas Contingency Operation, utilizado en las declaraciones públicas y en la justificación del gasto de las misiones o War Against Al Qaeda, presente en la National Security Strategy de 2010.

$4 \quad$ El concepto de Strategic Transition se ha popularizado en la jerga especializada - tras su aparición en la Strategic Defense Guidance (2012) para explicar la transformación de una arquitectura defensiva enfocada a los conflictos presentes a otra capaz de enfrentarse a los retos futuros. 
-marcada por la conclusión de la guerra contra el terror, la reorganización de la presencia avanzada, un creciente aislacionismo, la codificación de nuevos riesgos y la orientación de su arquitectura defensiva hacia los conflictos convencionalesha motivado que la Transformación, como herramienta para preparar la arquitectura militar para los conflictos futuros, domine la reflexión estratégica.

En segundo lugar, la transición entre cada uno de estos periodos está marcada por algún suceso -el principio del fin de la Guerra Fría, el trágico final de la posguerra fría o el desenlace de la guerra contra el terror- que la comunidad de defensa estadounidense percibe como la evidencia de que el modelo militar vigente ya no es el más adecuado para satisfacer las necesidades estratégicas del país. Esta situación facilita la consolidación y posterior popularización -durante la Guerra del Golfo, tras las operaciones de combate en Afganistán e Iraq o con la publicación de la Guía Estratégica de Defensa (Department of Defense [DoD], 2012)- de un nuevo modelo que se había estado gestando con anterioridad y que resuelve las carencias del paradigma anterior. Además, la transición entre cada una de estas fases no se relaciona necesariamente con un hecho violento, una alteración del equilibrio estratégico o un suceso de gran impacto militar; sino que se debe a la percepción de la comunidad de defensa estadounidense sobre la inadecuación de su arquitectura militar para satisfacer los retos emergentes.

La Tabla que se presenta a continuación expone gráficamente las tres etapas en las que se divide el pensamiento estratégico estadounidense desde el final de la Guerra Fría y los ejes sobre los que este se ha configurado:

$1991-2001$

\begin{tabular}{|c|c|c|c|}
\hline \multirow[b]{2}{*}{ Marco estratégico } & & \\
\hline & Pausa estratégica & Guerra contra el terror & Transición estratégica \\
\hline Punto de inflexión & Caída del Muro & & Eliminación de Osama \\
\hline Punto de intiexion & de Berlín & 11 septiembre 2001 & Bin Laden \\
\hline Consolidación & Guerra del Golfo & Afganistán e Iraq & $\begin{array}{l}\text { Guía Estratégica de la } \\
\text { Defensa }\end{array}$ \\
\hline $\begin{array}{l}\text { Paradigma } \\
\text { estratégico }\end{array}$ & $\begin{array}{l}\text { Revolución en los } \\
\text { Asuntos Militares }\end{array}$ & $\begin{array}{l}\text { Transformación (como } \\
\text { imperativo estratégico) }\end{array}$ & $\begin{array}{l}\text { Transformación (para } \\
\text { conflictos futuros) }\end{array}$ \\
\hline
\end{tabular}

Como se podrá observar a continuación, el pensamiento estratégico estadounidense ha experimentado varios cambios, pero siempre ha mantenido una gran continuidad. En este sentido, este parece haber seguido el esquema dialéctico hegeliano, ya que las distintas crisis que han revelado las limitaciones, contradicciones o inadecuación de su concepción militar no han motivado una rotura total con el modelo estratégico anterior, sino una síntesis del mismo, que resuelve los problemas identificados y facilita la consolidación de un nuevo paradigma militar.

Finalmente, antes de abordar el estudio es conveniente realizar dos breves comentarios de tipo conceptual y metodológico. Por un lado, el trabajo concebirá el pensamiento estratégico como un ejercicio racional, reflexivo, prescriptivo y anticipatorio que, basado 
en la identificación, codificación y priorización de cualquier evento susceptible de condicionar el entorno de seguridad, pretende fijar las líneas de acción más efectivas para resolver los problemas presentes y futuros (Coutau-Bégarie, 2003). Su ámbito de aplicación comprende desde la estrategia de seguridad nacional a la política militar y el empleo efectivo de la fuerza (Luttwak, 1987: 69-71). Asimismo, se entenderá que la política de defensa es la dimensión de la seguridad nacional encargada de establecer los fines, determinar los objetivos y proporcionar los medios necesarios para la defensa (Hays et al., 1997: 7-16). Su planeamiento se fundamenta en la concepción estratégica dominante y su elaboración requiere priorizar y conciliar factores geopolíticos, estratégicos, operativos, tecnológicos, económicos o domésticos. Por otro lado, la metodología utilizada para analizar tanto la evolución del pensamiento estratégico como la política de defensa estadounidense se fundamentará principalmente en la lectura de los documentos político-militares del país, las reflexiones de sus centros de estudios estratégicos y las publicaciones especializadas en la materia.

Después de exponer estas cuestiones previas, a continuación se procederá a estudiar la evolución del pensamiento estratégico estadounidense desde el final de la Guerra Fría hasta la actualidad.

\section{EL PENSAMIENTO ESTRATÉGICO EN LA POSGUERRA FRÍA}

El primer punto de inflexión en nuestra historia reciente se produce con la caída del Muro de Berlín el 9 de noviembre de 1989. Este suceso marcó el inicio del fin del orden internacional bipolar, catapultó a Estados Unidos a la cúspide del orden internacional, supuso el desvanecimiento de los dos grandes pilares estratégicos de la Guerra Fría -la Destrucción Mutua Asegurada ${ }^{5}$ y los grandes ejércitos convencionales- y comportó la reestructuración de las políticas de seguridad y defensa de los antiguos bloques (Eland, 2001).

No obstante, mientras desaparecía la amenaza sobre la que Washington había construido su arquitectura defensiva y la aparente estabilidad global permitía reducir su gasto militar y cobrar el dividendo de la paz, también empezaban a vislumbrarse nuevos peligros de distinta naturaleza y procedencia que aconsejaron ampliar el concepto de seguridad más allá de la tradicional defensa del territorio.

Y es que la amenaza de desatarse una Tercera Guerra Mundial, contemplada como factible hasta entonces, dejó paso a un mundo en el que se combinaban riesgos tan dispares como los estados fallidos, débiles o en descomposición, las catástrofes medioambientales, las migraciones incontroladas, las crisis humanitarias, la criminalidad transnacional o la proliferación de armamento de destrucción masiva. En otras palabras, el mundo bipolar

\footnotetext{
La Destrucción Mutua Asegurada puede definirse como un punto de equilibrio en el que un conflicto nuclear total entre Estados Unidos y la Unión Soviética comportaría la destrucción -estimada en 50-70\% del tejido industrial y 33-40\% de la población- de ambos contendientes. Esta situación se logró durante la década de 1960, cuando los arsenales atómicos de ambas potencias alcanzaron la paridad; sus fuerzas nucleares se organizaron en una tríada que garantizaba la capacidad de contragolpe y la disuasión unilateral dejó paso a la disuasión mutua.
} 
había sido sustituido por un orden internacional que pronto se revelaría muy distinto al imaginado por Francis Fukuyama en plena euforia poscomunista.

Mientras Estados Unidos estaba disfrutando de su pausa estratégica, en varios puntos del globo estallaron crisis que precisaron una respuesta de la comunidad internacional y revelaron algunos de los nuevos requerimientos que deberían satisfacer los ejércitos de la posguerra fría. No solo deberían estar en condiciones para responder a múltiples crisis que surgieran en cualquier punto del planeta; sino que una vez allí deberían realizar, de manera autónoma o en un entorno multinacional, una amplia variedad de actividades (asistencia humanitaria, interposición, estabilización o acciones convencionales); contra adversarios diversos (ejércitos regulares o fuerzas irregulares); en cualquier ambiente (urbano, montañoso, desierto o selvático) y en un marco más complejo y confuso que en el pasado, donde factores ajenos a los militares (legales, sociales, políticos, humanitarios, domésticos o mediáticos) no solo podrían condicionar el curso de la operación, sino también determinar su desenlace (Creveld, 2000).

En consecuencia, las fuerzas armadas estadounidenses -equipadas, organizadas y adiestradas para librar una guerra total en el continente europeo y el sudeste asiáticono solo debían prepararse para un mayor número de contingencias, sino que también tenían que reducir y reestructurar su potencial humano y militar para adaptarse a la disipación de la amenaza soviética, la desaceleración de su economía y el cobro del dividendo de la paz (Snider, 1993). ${ }^{6}$ Sin embargo, a pesar de la trascendencia de estos asuntos, durante la posguerra fría el análisis estratégico se articuló en torno a la crisis del vínculo trasatlántico, las nuevas concepciones de seguridad, las operaciones de paz, la privatización de la guerra y, sobre todo, la Revolución en los Asuntos Militares; y eran muy pocos los estrategas que se aventuraron a plantear seriamente cómo serían los conflictos futuros. ${ }^{7}$ En este sentido, es probable que la hegemonía americana, la aparente estabilidad internacional y la inexistencia de una amenaza concreta y definida motivaran el abandono del análisis estratégico y reforzaran la creencia de que el mundo había entrado en un periodo de paz que se dilataría hasta bien entrado el siglo XXI.

Por lo tanto, no es extraño que en este vacío estratégico la idea de una RMA motivada por la aplicación militar de las tecnologías de la información y capaz de transformar el arte de la guerra, articulara el debate estratégico estadounidense e internacional hasta $2001 .^{8}$

Originada tras la derrota norteamericana en Vietnam para terminar con el equilibrio del terror reinante en Europa durante los años setenta, estudiada en secreto en las más altas esferas de Washington y de Moscú durante los ochenta y popularizada mundialmente tras la espectacular victoria de la coalición liderada por Estados Unidos en la Guerra del

6 De hecho, estas temáticas constituyen los principios sobre los que se formuló la Base Force (1989-92), la primera gran revisión de la defensa estadounidense tras el fin de la Guerra Fría.

Véase, por ejemplo, Sullivan y Dubik (1995); Peters (1995) o Creveld (1991).

8 En términos generales, una RMA es una profunda transformación en la forma de combatir que resulta de la integración de nuevas tecnologías, conceptos operativos o formas de organización en las fuerzas armadas; que convierte en irrelevante u obsoleto el paradigma militar previo y proporciona una enorme superioridad al primer ejército que explota estas capacidades. Ello provoca que sus posibles adversarios deban alcanzar este nuevo estándar de capacidades, bien sumándose a la revolución o desarrollando respuestas susceptibles de acabar con esta ventaja (Colom, 2008). 
Golfo de 1991, esta revolución prometía victorias rápidas, decisivas y sin apenas daños colaterales en toda la gama de las operaciones gracias al empleo de fuerzas tecnificadas y con un pleno conocimiento del entorno operativo (Schneider y Grinter, 1998).

Estas características hacían de la RMA la solución perfecta a los problemas estratégicos que debían superar las sociedades avanzadas de fin de siglo: la erosión del modelo de ciudadano-soldado y el fin de la conscripción universal, la disminución del gasto militar, la creciente participación en operaciones de gestión de crisis, la necesidad de conservar la supremacía militar frente a adversarios futuros, y muy especialmente, parecía enmendar la creciente dificultad de las sociedades posheroicas para emplear la fuerza militar como herramienta política (Moskos et al., 2000).

En consecuencia, parece lógico que esta revolución sedujera a la clase política, militar, académica e industrial estadounidense. No solo prometía suplir la reducción de efectivos humanos, materiales y financieros con tecnología, sino que también brindaba a Washington la supremacía militar futura y la posibilidad de continuar empleando la fuerza armada como elemento de política exterior con unos costes políticos, económicos y sociales perfectamente asumibles por su opinión pública. ${ }^{9}$

Paralelamente, expertos de todo el globo se apresuraron en determinar las características fundamentales de esta revolución y sus potenciales efectos sobre el arte de la guerra. Así, observaron que su esencia radicaba en el sistema de sistemas o la capacidad que tendría cualquier sensor, plataforma, combatiente o arma para interactuar con el resto gracias a su integración en red. Ello proporcionaría un pleno conocimiento del campo de batalla y permitiría que una fuerza conjunta geográficamente dispersa batiera con plena precisión cualquier objetivo a gran distancia y sin apenas daños colaterales (Owens, 1995). Tal posibilidad sentaría las bases de la guerra en red, considerada como el estilo bélico propio de la Era de la Información (Alberts et al., 1999).

Y para alcanzar esta revolución que inauguraría un nuevo modelo americano de combatir (Boot, 2003), ${ }^{10}$ los estrategas juzgaron esencial adquirir las modernas tecnologías propias de la Era de la Información (plataformas furtivas, equipos informáticos, armamento inteligente o sistemas no tripulados integrados en red formando un sistema de sistemas) (Friedman, 1998); desarrollar nuevas formas de actuación (acción conjunta y combinada,

9 Sin embargo, en la inmediata posguerra fría la clase política americana estaba más preocupada en adaptar la arquitectura defensiva del país a la disipación de la amenaza soviética que en analizar las implicaciones de esta revolución que podía convertir en obsoletos muchos materiales del inventario militar del país. Aunque todavía faltarían unos años para que esta idea se consolidara entre la clase política del país, en 1993 el Secretario de Defensa Les Aspin ya empezó a plantear las posibilidades que brindaba la RMA para resolver ciertos problemas estratégicos que debía afrontar Estados Unidos en la posguerra fría, tales como mantener la estrategia de combatir en dos conflictos geográficamente alejados -en Oriente Medio y extremo Oriente-con una estructura de fuerzas menor que la proyectada durante la Guerra Fría (DoD, 1993).

10 Max Boot concibió el término New American Way of War para distinguirlo del estilo militar tradicional -de corte logístico y fundamentado en la capacidad industrial americana para derrotar al adversario mediante una guerra de desgaste (Weigley, 1977)- y detallar el estilo militar propio de la RMA, fundamentado este en la tecnología, el conocimiento y la precisión para lograr victorias rápidas, limpias y contundentes frente a cualquier adversario. Aunque las campañas afgana e iraquí revelaron sus limitaciones, el creciente empleo de Drones (sistemas no tripulados de ataque), armamento de precisión y fuerzas de operaciones especiales sugieren que este modelo está acomodándose a los ejes del pensamiento estratégico actual. 
vocación expedicionaria y operaciones espaciales, ciberespaciales e informativas); aplicar nuevas formas de organización (simplificación de estructuras, generación de fuerzas modulares, proyectables y preparadas para actuar en todo el espectro de las operaciones); implantar nuevas competencias e implementar nuevas formas de conducir las operaciones militares (descentralización del mando, nueva organización de los estados mayores, mayor control estratégico y político de las operaciones o integración de la cadena de mando militar bajo control civil en operaciones interagencia) (Lippitz y White, 2000). Estos cambios en la estructura, equipamiento, organización y funcionamiento de las fuerzas armadas para conquistar la RMA pasaron a fundamentar el planeamiento de la defensa estadounidense durante la década de los noventa (Kagan, 2006).

Resumiendo, mientras el estudio de la Revolución en los Asuntos Militares, la identificación de sus características definidoras y la evaluación de sus potenciales efectos constituyeron los ejes sobre los que se articuló el pensamiento estratégico estadounidense durante la posguerra fría; el logro de la RMA y la generación de un nuevo catálogo de capacidades aplicable a toda la gama de operaciones presentes y futuras lo hicieron sobre su planeamiento de la defensa. ${ }^{11}$ Este proceso enfocado a lograr la revolución y desarrollar un conjunto de capacidades militares adecuadas para las guerras futuras recibiría el nombre de Transformación (DoD, 1997). No obstante, debería esperarse hasta septiembre de 2001 para que este proceso se convirtiera en un imperativo estratégico para adaptar la maquinaria militar estadounidense al mundo del tercer milenio (DoD, 2001 y Rumsfeld, 2002).

En conclusión, durante este periodo el pensamiento estratégico y el planeamiento de la defensa americanos se articularon en torno a la RMA, una revolución que parecía constituir la respuesta a los interrogantes estratégicos que debía responder Estados Unidos a finales del siglo XX. Posibilitada por las tecnologías de la información, fundamentada en la obtención del pleno conocimiento del campo de batalla y configurada en torno a la generación de pequeñas fuerzas conjunto-combinadas dominando las esferas terrestre, marítima, aérea, espacial, ciberespacial e informativa para lograr victorias rápidas, limpias, decisivas y sin apenas daños colaterales, esta RMA parecía ser la culminación de la guerra convencional (Colom, 2008).

Y es que si bien esta revolución pretendía confeccionar un catálogo de capacidades militares apropiado para actuar en toda la gama de operaciones y derrotar a cualquier adversario presente o futuro, en general se asumía que esta permitiría incrementar la brecha convencional entre Estados Unidos y cualquiera de sus posibles competidores futuros. En este período de fervor revolucionario, fueron muy pocos los estrategas que alertaron de la excesiva confianza puesta en la tecnología, las posibles limitaciones de la RMA en ambientes irregulares o las incógnitas que suscitaba este nuevo estilo

11 Para conocer con más detalle el alcance de esta afirmación, pueden observarse los principales documentos estratégicos y de planeamiento realizados por el Pentágono (especialmente la Quadrennial Defense Review de 1997); los informes anuales del Secretario de Defensa durante el periodo 1995-2001, los trabajos de la Junta de Jefes de Estado Mayor (en particular las Joint Vision de 1996 y 2000), los libros blancos de los tres ejércitos o el informe Transforming Defense: National Security in the $21^{\text {st }}$ Century, elaborado en 1997 por un grupo de expertos a petición del Pentágono para responder a los interrogantes estratégicos del país. 
de combatir a tenor de las experiencias en Somalia o los Balcanes. Convencidos de que cualquier competidor de Estados Unidos intentaría conquistar esta revolución, solamente tomaron en consideración la respuesta asimétrica planteada por dos oficiales de la República Popular China y que Washington asumió como una postura oficiosa de Beijín (Flanagan y Marti, 2003).

Y es que tras proclamar públicamente que el logro de una Revolución en los Asuntos Militares con características chinas ${ }^{12}$ debía fundamentar el planeamiento estratégico y militar chino para el siglo XXI y de reconocer que el país carecía de los medios económicos, tecnológicos, industriales ni militares para disputar a Estados Unidos el liderazgo de la RMA, estos dos oficiales propusieron una posible -y controvertida- solución: emplear acciones de guerra sin restricciones (empleo de armamento de destrucción masiva, terrorismo indiscriminado, ciberguerra, disrupción de los flujos financieros y las redes de información y comunicaciones, manipulación de las opiniones públicas o guerra legal) para anular la supremacía militar estadounidense (Liang y Xiangsui, 2004). Aunque estos planteamientos han sido reiteradamente negados por las autoridades chinas, desde la década de 1990 Beijín está desarrollando medidas específicamente diseñadas para explotar las vulnerabilidades de Estados Unidos y de su estilo militar (DoD, 2011). ${ }^{13}$

Sin embargo, en este periodo marcado por una excesiva confianza en la tecnología, prácticamente ningún estratega podía imaginar que una forma de lucha a priori tan arcaica y simple como la guerra irregular revelaría los límites del nuevo estilo militar americano (Henrotin, 2008).

Posiblemente, si los estrategas estadounidenses no hubieran puesto tantas confianzas en la tecnología y hubieran observado con mayor atención la evolución del entorno de seguridad global y la naturaleza de los conflictos que habían surgido tras la caída del Telón de Acero, con toda probabilidad estos habrían moderado sus proclamas revolucionarias y no se habrían visto sorprendidos por los acontecimientos de Afganistán o Iraq, como finalmente sucedió pocos años después.

\section{EL IMPERATIVO ESTRATÉGICO Y GUERRA CONTRA EL TERROR}

Los ataques terroristas del 11 de septiembre de 2001 contra Nueva York y Washington constituyen el segundo hito de nuestra historia reciente. Estos sucesos acabaron con la pausa estratégica iniciada tras la caída del Telón de Acero, revelaron algunos de

12 Planteada por el presidente Jiang Zemin en 1993, la Revolución china en los Asuntos Militares pretende mecanizar, profesionalizar e informatizar sus fuerzas armadas, reducir la brecha militar con Estados Unidos y estar en condiciones de triunfar en guerras locales en ambientes de alta tecnología que deben plasmarse en campañas cortas, decisivas y limitadas en sus vertientes geográfica (para combatir solamente en el área de influencia china), política (para garantizar la defensa de la seguridad y los intereses nacionales del país) y temporal (para no alterar el normal funcionamiento del país ni comprometer su crecimiento económico) (Ministry of National Defense, 2010).

13 Entre las medidas que Beijín está desarrollando para explotar las vulnerabilidades de Estados Unidos, destacan especialmente las armas antisatélites y las capacidades para combatir en el ciberespacio y en la esfera de la información. 
los nuevos riesgos y amenazas a la seguridad internacional y acallaron las proclamas revolucionarias de la década anterior.

Aunque esta segunda etapa arranca formalmente con el 11-S, sus fundamentos se habían establecido varios meses antes, coincidiendo con la llegada de George W. Bush a la Casa Blanca. Cautivado por el potencial de la RMA y consciente del papel que esta tendría en la configuración del nuevo orden mundial, ${ }^{14}$ el presidente Bush y su secretario de Defensa Rumsfeld trazaron un ambicioso proceso de Transformación para conquistar esta ansiada Revolución en los Asuntos Militares y preparar la arquitectura de defensa norteamericana para afrontar los retos emergentes. Para ello no solo se dispusieron a formular una política de seguridad, defensa y militar que acabara definitivamente con el paradigma de la Guerra Fría, sino que también situaron la transformación del conjunto de la defensa nacional -desde la estructura, volumen, equipamiento, capacidades y patrones de despliegue de las fuerzas armadas hasta la organización, funcionamiento, administración y financiación del Pentágono-como una de las principales prioridades políticas de la nueva administración americana (DoD, 2001 y 2003; Rumsfeld, 2002 o Cimbala, 2010).

No obstante, pronto la Transformación reemplazó a la RMA como foco del análisis estratégico internacional y el pilar del planeamiento de la defensa estadounidense. Los trágicos sucesos del 11 de septiembre de 2001 acabaron con la pausa estratégica iniciada tras la caída del Telón de Acero, convencieron a Washington de la urgencia de acomodar su arquitectura de defensa -una enorme, rígida y burocratizada estructura todavía diseñada para combatir en una guerra total contra la Unión Soviética- a la realidad del siglo XXI, aceleraron su transformación y le permitieron poner a prueba la revolución.

El bautismo de fuego de este nuevo estilo militar tuvo lugar en Afganistán, donde una pequeña fuerza creada ad hoc para la misión, apoyada permanentemente desde el aire y por fuerzas locales, equipada con sofisticadas tecnologías y usando avanzadas tácticas derrocó al régimen Talibán e instauró un gobierno de transición afín a Occidente en poco más de un mes. Esta contundente victoria sorprendió a la comunidad de defensa norteamericana, que no dudó en afirmar que la forma en que se habían desarrollado las operaciones eran signos inequívocos de que la RMA estaba muy cerca, por lo que propusieron acelerar el proceso de transformación. ${ }^{15}$

14 Aunque esta convicción puede observarse en varias declaraciones públicas, es especialmente significativo su primer discurso preelectoral en materia de seguridad. Realizado en la Academia Militar de Charleston (Carolina del Sur) el 23 de noviembre de 1999, el entonces candidato a la presidencia declaró que: ...My third goal is to take advantage of a tremendous opportunity to extend the current peace into the far realm of the future. A chance to project America's peaceful influence, not just across the world, but across the years. This opportunity is created by a revolution in the technology of war. [...] This revolution perfectly matches the strengths of our country-the skill of our people and the superiority of our technology. The best way to keep the peace is to redefine war on our terms. Yet today our military is still organized more for Cold War threats than for the challenges of a new century [...] Now we must shape the future with new concepts, new strategies, new resolve [...] As president, I will begin an immediate, comprehensive review of our military- the structure of its forces, the state of its strategy, the priorities of its procurement-conducted by a leadership team under the Secretary of Defense. I will give the Secretary a broad mandate- to challenge the status quo and envision a new architecture of American defense for decades to come.

15 Este sentir también existía entre la clase política americana, como lo atestigua el discurso de Bush en la Academia Militar de Charleston el 11 de diciembre de 2001 o las palabras de Rumsfeld en la Universidad Nacional de la Defensa el 31 de enero de 2001. 
A los pocos meses, Washington inició los preparativos para invadir Iraq. Dispuesto a superar las inercias históricas y acabar con la restrictiva doctrina Weinberger-Powell ${ }^{16}$ el Pentágono desarrolló un sofisticado plan de operaciones que permitiera explotar la RMA y experimentar con la transformación. ${ }^{17}$ Tras un breve despliegue y concentración de fuerzas, un destacamento conjunto terrestre-anfibio con permanente apoyo aéreo paralizó el régimen iraquí, causó una total confusión entre las filas de sus fuerzas armadas, anuló toda oposición militar digna de mención y logró un triunfo fulminante, aplastante y decisivo en cuestión de semanas. ${ }^{18}$

Estas espectaculares victorias parecían corroborar los frutos de la revolución, la buena marcha de la transformación y la eficacia del nuevo estilo militar americano. No obstante, entre las razones de estos éxitos se hallaban las semillas de los posteriores fracasos, ya que con el paso de las acciones de combate a las labores de estabilización, factores como la reducida entidad de la fuerzas desplegadas, los medios materiales empleados, las carencias doctrinales en materia de seguridad, estabilización, reconstrucción o antiterrorismo, el desconocimiento de ambas culturas, la insuficiente inteligencia humana de la que disponían o el férreo control de las operaciones desde unos estados mayores localizados a miles de kilómetros; unidos estos a la inexistencia de un plan coherente para su pacificación y las erróneas decisiones que se tomaron al finalizar las operaciones de combate, facilitaron que en ambos escenarios estallara rápidamente una insurgencia que ha permanecido activa hasta hoy a pesar de los enormes esfuerzos de la comunidad internacional para estabilizar el territorio (Kagan, 2006).

El estallido de la insurgencia cogió -tal y como había sucedido hace cuarenta años en Vietnam-desprevenida al grueso de la comunidad estratégica americana ${ }^{19}$ que, fascinada por las posibilidades de la RMA, parecía haber obviado algunos de los fundamentos de la guerra, en particular que esta es siempre un choque de voluntades y que cualquier actor busca explotar las debilidades del adversario, lucha con los medios que tiene a su alcance y emplea las estrategias que mayores beneficios le proporciona. Así, frente a la supremacía convencional del invasor, la resistencia afgana e iraquí planteó respuestas

16 Planteada por el secretario de Defensa Caspar Weinberger en 1984 y refinada por el jefe de la Junta de Jefes de Estado Mayor Colin Powell en 1991, esta doctrina imponía severas limitaciones al empleo de la fuerza armada para evitar otro Vietnam. Para ello, recomendaba que esta se utilizara como último recurso, de manera aplastante y con objetivos estratégicos y fines políticos claramente definidos.

17 No obstante, la elaboración de este plan de operaciones no fue exento de controversias entre Rumsfeld y la cúpula militar en relación con la estrategia a seguir y el volumen de fuerzas a emplear en la invasión de Iraq. De hecho, el intervencionismo en la planificación y la microgestión de las operaciones realizada por el titular de Defensa motivaron que Eliot Cohen (2003: 225-40) afirmara que Rumsfeld acabó con el modelo de relaciones civiles-militares impuesto en el país tras la debacle de Vietnam.

18 Tales afirmaciones pueden observarse en las comparecencias del secretario Rumsfeld y el general Tommy Franks -comandante de la fuerza multinacional en Iraq- ante el Comité del Senado para las Fuerzas Armadas el 9 de julio de 2003 o el discurso del subsecretario de Defensa Paul Wolfowitz en la Escuela de Guerra Naval el 20 de junio de 2003.

19 La única excepción destacable radicaba en los civiles y militares vinculados con las operaciones especiales que, tradicionalmente interesados por las Small Wars, se habían alejado del debate sobre la RMA. A partir de entonces, esta comunidad no solo orientaría los debates teóricos y realizaría importantes aportaciones prácticas, sino que también incrementaría su presencia militar e influencia política en el Pentágono. 
que aprovecharan las lagunas del estilo militar estadounidense y explotaran las vulnerabilidades del país. ${ }^{20}$

La violencia insurgente no solo puso de manifiesto las limitaciones de la RMA en escenarios irregulares, sino también las dificultades políticas, estratégicas y operativas que entraña la estabilización de zonas hostiles, los costes humanos, materiales y políticos que conllevan los cambios de régimen por la fuerza o las nuevas y apremiantes necesidades operativas motivadas por la participación militar estadounidense en ambos conflictos. Estos factores motivaron la desaparición definitiva de la revolución de la agenda política y militar estadounidense; un cambio de rumbo de la transformación -de prepararse para las guerras futuras a resolver los problemas presentes- que los ejércitos apadrinaron inmediatamente, pero que no se formalizó políticamente hasta el año $2006^{21}$ y la orientación del pensamiento estratégico americano hacia los conflictos irregulares y las insurgencias (Kilcullen, 2009).

En esta coyuntura, la comunidad de defensa americana se volcó en apoyar a los ejércitos para resolver la situación: rescató los conceptos de conflicto irregular (contrario a los usos y costumbres de la guerra) y conflicto asimétrico (orientado a explotar las vulnerabilidades de las fuerzas regulares) para definir las amenazas que se cernían sobre las fuerzas armadas y los principios de la contrainsurgencia clásica para contener la violencia insurgente; ayudó a reescribir las doctrinas de estabilización y reconstrucción, cooperación civil-militar o apoyo a las autoridades civiles; buscó respuestas para los retos de la construcción nacional e intentó descubrir la naturaleza e implicaciones de la guerra irregular en el siglo XXI. ${ }^{22}$

20 Entre estas debilidades cabe destacar la volubilidad de la opinión pública doméstica y la presión de la comunidad internacional; el pánico a las bajas propias y el temor a los daños colaterales; el sometimiento a unos usos y costumbres de la guerra restrictivos y anacrónicos; la ansiedad por los costes políticos y efectos electorales de las operaciones; la exigencia de restringir su alcance, impacto y duración; la reticencia a utilizar fuerzas terrestres en operaciones o la necesidad de emplear la fuerza de forma limitada y restrictiva.

21 No fue hasta la presentación de la Quadrennial Defense Review de 2006 -que establecía las líneas maestras de la defensa nacional del segundo mandato de Bush-cuando este enfoque se formalizó políticamente. Sin embargo, fue preciso esperar hasta la dimisión de Donald Rumsfeld y el nombramiento de Robert Gates como titular de Defensa para que esta nueva orientación empezara a materializarse mediante ajustes en el planeamiento de defensa (priorizando el desarrollo de capacidades aptas para las operaciones en curso), en los programas de obtención de material (redefiniendo o posponiendo los grandes proyectos de armamento futuros y adquiriendo por procedimiento de urgencia los equipos necesarios para las campañas actuales) y en la estructura de fuerzas (reconvirtiendo grupos de artillería en unidades de infantería, incrementando el número de fuerzas de operaciones especiales, unidades de cooperación civil-militar, replanteando los ciclos de despliegue o regulando la presencia de contratistas militares privados).

22 Durante este periodo caracterizado por una estrecha colaboración civil-militar -y con carácter previo a la consolidación institucional del cambio de rumbo de la Transformación-, las fuerzas armadas procedieron a mejorar sus competencias culturales y lingüísticas; prepararse para realizar labores de estabilización, apoyo a la reconstrucción, contrainsurgencia, contraterrorismo o seguridad; incrementar la inteligencia humana sobre el terreno; reforzar la cooperación civil-militar y adquirir, con la mayor celeridad posible y utilizando los recursos propios, los materiales necesarios para las operaciones en curso. En el plano operativo, en 2002 ya se experimentó con los Provincial Reconstruction Teams -herederos de los Civil Operations and Rural Development Support empleados en Vietnam para combatir a la insurgencia- para combinar las labores de reconstrucción y contrainsurgencia en Afganistán, y cinco años después se empleó la Surge -una estrategia fundamentada en el cierre del teatro de operaciones, el incremento de las fuerzas de combate empleadas en contrainsurgencia y el aislamiento de los elementos insurgentes más extremistas- con gran éxito en Iraq para reducir la violencia política y avanzar en la estabilización del país. Adaptada con más o menos fortuna al escenario afgano, esta estrategia fue utilizada por Obama en 2009 para intentar estabilizar el país y sentar las precondiciones necesarias para la transferencia de autoridad al gobierno afgano. 
Tras numerosos debates y varios intentos fallidos, a mediados de la década se fraguó la guerra híbrida, un concepto que pronto lograría imponerse entre la comunidad de defensa estadounidense para detallar esta forma de lucha que parecía ser una evolución de la guerra irregular o asimétrica tradicional. Definido originalmente en 2002 para advertir de las tácticas empleadas por la insurgencia chechena contra el ejército ruso durante la Primera Guerra de Chechenia, este término fue empleado por primera vez de forma oficial en la Estrategia Nacional de Defensa de 2005 para explicar la combinación de dos o más amenazas de tipo tradicional, irregular, catastrófico o disruptivo (DoD, 2005). Sin embargo, no fue hasta la guerra del verano de 2006 entre Israel y Hezbollah cuando este concepto se popularizó hasta convertirse en uno de los ejes del debate sobre la transformación de la guerra. ${ }^{23}$

Concebido como un estilo de lucha característico del mundo globalizado y la respuesta lógica al paradigma militar estadounidense, la guerra híbrida se define por "...el empleo de cualquier modo de lucha disponible, incluyendo formas convencionales, tácticas irregulares, actos terroristas indiscriminados e incluso actividades criminales" (Hoffman, 2007: 8). A este rasgo se le añaden otras características como el empleo de armamento sofisticado, sistemas de uso dual o armas sencillas y asequibles usadas de forma novedosa; la explotación de las tecnologías de la información (sistemas de posicionamiento, Internet o telefonía móvil) para obtener inteligencia, garantizar el mando y control de las operaciones, difundir eficazmente su mensaje político o planear ciberataques contra las redes civiles y militares del adversario; una organización interna flexible, adaptable y articulada en red; su completa indefinición normativa y total desprecio a los usos y costumbres de la guerra tradicionalmente aceptados por la comunidad internacional; la variedad de fuentes de financiación manejadas (actividades legales y delictivas en estrecha colaboración con el crimen organizado) o el eficaz empleo de todos los medios que estén a su disposición para infligir el máximo daño a su adversario. Este híbrido entre la lucha convencional e irregular puede ser empleado tanto por Estados como por actores no estatales y su difusión impondrá nuevos retos a las fuerzas armadas en operaciones.

Con independencia de si la guerra híbrida es algo totalmente nuevo o, por el contrario, constituye una evolución de la guerra irregular adaptada al mundo actual, este concepto ha logrado hacerse un lugar en el pensamiento estratégico norteamericano ${ }^{24}$ para exponer la complejidad de los conflictos actuales y la difuminación de la frontera entre lo regular y lo irregular; ilustrar sobre los peligros que plantean las formas de guerra asimétrica sobre unos ejércitos que todavía no han superado el paradigma bélico de la Guerra Fría

23 Además del estudio de la literatura existente, para conocer la construcción conceptual de la guerra híbrida es imprescindible repasar los debates virtuales que todavía continúan en los foros de la revista electrónica Small Wars Journal (www.smallwarsjournal.com).

24 Los conceptos de guerra o amenaza híbrida son ampliamente utilizados en las declaraciones políticas y militares del país y han sido incluidas en sus principales documentos estratégicos, entre los que destacan la National Security Strategy (2010); la Quadrennial Defense Review (2010) o el Capstone Concept for Joint Operations (2012). Sin embargo, no se considera que la guerra híbrida constituya una nueva forma de guerra. En otras palabras: Senior military officials in recent public testimony asserted the increased likelihood of U.S. forces encountering an adversary that uses hybrid warfare tactics, techniques, and procedures. However, DoD has not officially defined hybrid warfare at this time and has no plans to do so because DoD does not consider it a new form of warfare (Government Accounting Office, 2010: 2). 
y favorecer un proceso de Transformación que proporcione a las fuerzas armadas del país las capacidades requeridas para enfrentarse a cualquier adversario, en cualquier ambiente y en toda la gama de las operaciones.

En resumen, el 11 de septiembre de 2001 terminó con la pausa estratégica surgida tras el fin del mundo bipolar y liquidó el modelo de seguridad y defensa vigente hasta entonces. Estos hechos convirtieron a la Transformación en un imperativo estratégico y en la base del planeamiento de la defensa estadounidense en detrimento de la RMA, que fue relegada a un segundo plano. Sin embargo, el canto del cisne de esta revolución se produjo poco después, cuando las espectaculares victorias logradas por la coalición liderada por Estados Unidos en la invasión de Afganistán e Iraq mostraron al mundo las cualidades de este estilo de lucha que prometía triunfos rápidos, limpios y decisivos mediante el empleo de una fuerza precisa y abrumadora. No obstante, el comienzo de las labores de estabilización y reconstrucción junto con el estallido de la insurgencia revelaron las limitaciones de este estilo militar. Este baño de realismo evidenció las carencias de unas fuerzas armadas todavía ancladas en el paradigma de la Guerra Fría, mostró algunas de las nuevas amenazas que se cernían sobre los ejércitos y expuso las limitaciones de una Transformación excesivamente tecnocéntrica y centrada en la lucha convencional. Estos factores acabaron con el furor revolucionario de los noventa, desterraron la RMA del análisis estratégico y motivaron un cambio de rumbo en la Transformación para contribuir a la guerra contra el terror.

Este giro estratégico provocó que el planeamiento de la defensa estadounidense pasara a articularse en torno a la generación de capacidades para la estabilización posconflicto, la construcción nacional o la lucha contra la insurgencia; y en el pensamiento estratégico primara el estudio de las amenazas irregulares e híbridas, consideradas por muchos como el mayor peligro que se cernía sobre los ejércitos regulares avanzados. No obstante, el renovado interés por la guerra asimétrica, el conflicto de baja intensidad, el planeamiento de la defensa de contingencia y, tras la victoria de Barack H. Obama, por los controvertidos Drones (sistemas aéreos no tripulados de ataque), relegaron a un puesto secundario otros riesgos más tradicionales -el peligro de un Pakistán fallido, el arma atómica norcoreana, la proliferación persa, el expansionismo chino o la seguridad de los suministros energéticos- que estaban surgiendo durante esta misma etapa y que no se integrarían en el pensamiento estratégico estadounidense hasta la muerte de Osama Bin Laden y el cierre de facto de la guerra contra el terror.

\section{EL PENSAMIENTO ESTRATÉGICO EN UN MUNDO INCIERTO}

El tercer y último punto de inflexión en nuestra historia reciente se sitúa el 2 de mayo de 2011, cuando la eliminación de Osama Bin Laden terminó de facto con la guerra contra el terror, ${ }^{25}$ permitió adelantar los repliegues de Iraq (2011) y Afganistán (2014) y

25 No obstante, Al Qaeda y sus organizaciones afines continúan siendo calificadas por Washington como una amenaza, como lo atestigua la Strategic Defense Guidance (2012) y los comentarios vertidos durante los debates presidenciales para los comicios de 2012. 
facilitó la sustitución del agotado modelo de seguridad y defensa vigente -enfocado a la construcción de estados, la insurgencia y el contraterrorismo- por un nuevo paradigma que, motivado por la coyuntura doméstica e internacional, se orienta al mantenimiento de la supremacía militar frente a cualquier adversario futuro. Gestado en los años anteriores, este modelo se consolidó a principios de 2012, cuando el presidente Obama presentó la Guía Estratégica de la Defensa, una hoja de ruta que establece las líneas maestras de la política de defensa y la organización militar para los próximos años (DoD, 2012). ${ }^{26}$

Aunque Al Qaeda no ha sido totalmente derrotada y la inestabilidad del norte de África brinda nuevas oportunidades a esta organización, la salida de Iraq ha dejado al país en una delicada situación y el repliegue de Afganistán se ejecutará sin haber logrado la situación final deseada; la sensación de amenaza que surgió tras el 11-S se ha debilitado, la esperanza de normalizar la situación de estos dos países se ha desvanecido, el panorama estratégico se ha transformado, Estados Unidos ha dejado de ser el único polo del poder global y la crisis económica requiere redefinir a la baja los objetivos de fuerzas, catálogos de capacidades, planes de modernización o patrones de despliegue y priorizar el menguante gasto militar. ${ }^{27}$

En consecuencia, los estrategas del país han dado carpetazo a todo lo relacionado con la guerra contra el terror y han vuelto a interesarse por otros riesgos y amenazas más tradicionales. Así, las grandes contingencias de la pasada década -aunque limitadas e incapaces de alterar la estructura del sistema internacional- como la guerra contra el terror, la construcción de estados o la insurgencia han dejado paso a otros peligros susceptibles de perturbar el equilibrio estratégico, como la proliferación de armamento de destrucción masiva, la inestabilidad del mundo árabe y musulmán, la competición entre los poderes emergentes y las potencias consolidadas por la hegemonía regional y el control de los recursos, la geopolítica del escenario Asia-Pacífico y las ambiciones del gigante chino, la carrera armamentística del lejano Oriente o las amenazas que se ciernen sobre el libre acceso a los bienes comunes globales como los mares, el cielo, el espacio, el ciberespacio o la esfera de la información (Denmark y Mulvenon, 2010).

Además, es ahora cuando las lecciones aprendidas de la guerra contra el terror se están integrando en el pensamiento estratégico estadounidense. Así, desde una óptica política se ha comprendido la extrema dificultad que presenta una democracia avanzada para emplear la fuerza armada en defensa de su interés nacional y la inviabilidad práctica de mantener largas campañas militares. También se ha expuesto la volubilidad de la opinión pública, la influencia de los medios de comunicación de masas para condicionar la acción política, la incompatibilidad entre los ciclos políticos y las opciones estratégicas,

26 Aunque este documento carece de valor legislativo - es una hoja de ruta que elaboró el ejecutivo ad hoc para presentar un plan de ajuste previo al debate sobre los presupuestos de 2013 y así bloquear la acción de la Cámara baja, controlada por el Partido Republicano- es muy probable que sus contenidos se formalicen en la National Security Strategy y la Quadrennial Defense Review que el ejecutivo elaborará a lo largo del próximo año. En 2011 se aprobó el Budget Control Act of 2011 (Public Law 112-25-S.365, de 2 de agosto de 2011) que reducía la base de gasto militar en 487.000 millones de dólares en los próximos diez años, una cifra que podría doblarse en caso de no lograr la contención del déficit público. Aunque la oposición ha sido muy crítica con esta norma, la reelección de Obama garantiza su implementación en los sucesivos ejercicios presupuestarios. 
las limitaciones del jus in bello en los conflictos actuales, la vigencia de la doctrina Weinberger-Powell para guiar la acción militar estadounidense, la peligrosidad que entrañan los cambios de régimen por la fuerza o la inviabilidad práctica de las labores de construcción nacional (Gvosdev y Reveron, 2010).

Desde una óptica militar, aunque las campañas afgana e iraquí han evidenciado la imbatibilidad de Estados Unidos en el terreno convencional, también han supuesto un baño de realismo que ha terminado con las proclamas revolucionarias de los noventa y obligado a replantear tanto el pensamiento estratégico como la política de defensa del país. Además, esta etapa ha expuesto las carencias del nuevo estilo norteamericano de combatir, orientado este al logro de victorias rápidas, contundentes y sin apenas daños colaterales mediante el empleo de una fuerza abrumadora y quirúrgica. Pero sobre todo, este periodo ha recordado a Washington las limitaciones de la tecnología y la incapacidad de ver lo que sucede "en el otro lado de la colina", acabado con la ilusión de las guerras limpias y servido para ratificar la inmutable naturaleza de la guerra, en la que la violencia, la destrucción y la muerte son sus elementos definidores; y recordar que la fuerza armada es la última razón del gobernante, y por ello debe ser utilizada como último recurso, de forma racional y orientada a la consecución de unos fines políticos claramente definidos, realistas y alcanzables en tiempo, espacio y forma.

Todos estos elementos han mediado para que Washington vuelva a enfocar su defensa hacia los riesgos convencionales y oriente su planteamiento estratégico hacia los conflictos regulares sin descuidar los híbridos. Ello ha motivado que Estados Unidos reduzca la presencia avanzada -entendida esta como el mantenimiento de fuerzas militares desplegadas permanentemente fuera del territorio estadounidense- y la concentre en la región Asia-Pacífico; abandone los despliegues de fuerzas masivos, descarte realizar grandes campañas militares o embarcarse en operaciones de cambio de régimen y construcción nacional o renuncie a conducir labores de estabilización, apoyo a la reconstrucción o lucha contra la insurgencia. Estas actividades están siendo sustituidas por un progresivo repliegue global y la priorización de la inteligencia prospectiva, el ataque estratégico, la proyección global y la capacidad de acceso a cualquier punto del planeta; las colaboraciones puntuales con terceros países; las operaciones limitadas en tiempo, espacio y medios implicados o las acciones contraterroristas puntuales con drones y fuerzas de operaciones especiales (DoD, 2012 y Tomes, 2012). El curso de los acontecimientos determinará la manera en que se refinan, consolidan y ejecutan estos nuevos principios estratégicos.

En conclusión, en los albores de esta nueva etapa, Estados Unidos no solo debe superar la herencia de la guerra contra el terror mientras refina un cuerpo de pensamiento adecuado a este periodo marcado por la escasez de recursos, la incertidumbre estratégica, el auge y consolidación de nuevas potencias y el retraimiento del país de los asuntos mundiales, sino también debe redefinir su espacio geoestratégico, examinar las tendencias globales para anticipar los retos emergentes y desarrollar nuevas ideas, conceptos y planteamientos que le permitan perfilar y generar las fuerzas armadas que necesitará para el día de mañana. Aunque es imposible prever la evolución, duración y ocaso de esta tercera marcada por una Transformación encaminada a anticipar la defensa estadounidense a 
los conflictos futuros, es ahora cuando está en juego la supremacía militar y hegemonía política del país en el mundo del siglo XXI.

\section{CONCLUSIONES}

Como el lector habrá observado a lo largo del trabajo, desde el final de la Guerra Fría el pensamiento estratégico estadounidense ha pasado por tres grandes periodos: la posguerra fría (1989-2001), la guerra contra el terror (2001-11) y la actualidad (2011-). Cada una de estas etapas ha estado condicionada por varios elementos externos que la enmarcan -el final del orden internacional bipolar, los ataques sobre Nueva York y Washington o la eliminación de Osama Bin Laden- y por otros factores internos que han condicionado y fundamentado el pensamiento estratégico: la adaptación de la arquitectura defensiva del país a la posguerra fría y la conquista de la Revolución en los Asuntos Militares; la Transformación como imperativo estratégico y para resolver los problemas observados en Afganistán e Iraq o la Transformación en un contexto de incertidumbre, crisis económica y transición estratégica para anticiparse a los conflictos futuros.

No obstante, a pesar de los cambios en el panorama global, en el entorno de amenazas o en la política doméstica estadounidense desde la caída del Telón de Acero, la transformación de su pensamiento estratégico se ha producido sin grandes fracturas, de forma evolucionista y sintetizando los hitos del periodo anterior con las nuevas ideas y planteamientos que iban consolidándose en cada momento.

Mientras la primera etapa estuvo marcada por la euforia revolucionaria y la creencia -tan presente en la mentalidad político-militar estadounidense- de que la tecnología permitiría resolver los interrogantes estratégicos del país y contribuiría a mantener su hegemonía política en el tercer milenio; en la segunda etapa se observaron las severas limitaciones del nuevo modelo militar americano y se conocieron los inmensos costes que entrañan los cambios de régimen por la fuerza y la construcción de nuevos estados obviando su bagaje cultural e histórico. Hoy se está consolidando un nuevo paradigma estratégico que, formalmente articulado en oposición al que reinó durante la guerra contra el terror, contiene las lecciones que Estados Unidos ha aprendido tras once años de guerra y parece fundamentarse -tal y como sugiere la limitada participación del país en el conflicto libio, su pasividad ante la guerra civil siria, la expansión geográfica de los ataques con drones, la reducción de la presencia militar en suelo europeo o la consolidación de la doctrina de batalla aeronaval para garantizar que las fuerzas navales y aéreas del país puedan acceder libremente a cualquier punto del globo- en un recogimiento estratégico, la limitación del volumen, alcance e impacto de sus acciones militares, la resistencia a emplear fuerzas terrestres en operaciones, la negativa a participar en labores de estabilización, apoyo militar a la reconstrucción o contrainsurgencia, la búsqueda de soluciones tecnológicas a los nuevos problemas estratégicos o la reorientación de su presencia avanzada hacia la región Asia-Pacífico con el fin de contener la expansión china y prepararse para un hipotético conflicto entre ambas potencias. En otras palabras, aunque el pensamiento estratégico y la política de defensa estadounidense se basan 
formalmente en la Transformación como solución a la incertidumbre y herramienta de anticipación a los conflictos futuros, esta va pareciéndose cada vez más al modelo tecnocéntrico de la Revolución en los Asuntos Militares.

Queda por ver cómo evolucionará, cuándo se consolidará y cuáles serán las razones que motivarán la crisis de este nuevo paradigma estratégico que empieza a recordar demasiado al que se impuso en Estados Unidos en la inmediata posguerra fría y que pronto quedó superado por la Historia.

\section{REFERENCIAS}

Bensahel, Nora. 2006. "Preventing Insurgencies after Major Combat Operations". Defence Studies 6 (3): 278-91. Boot, Max. 2003. "The New American Way of War". Foreign Affairs 82 (4): 41-58.

Cimbala, Stephen (ed.). 2010. The George W. Bush Defense Program: Policy, Strategy, and War. Washington DC: Potomac Books.

Cohen, Eliot. 2002. Supreme Command: Soldiers, Statesmen, and Leadership in Wartime. Nueva York: Anchor Books.

Colom, Guillem. 2008. Entre Ares y Atenea, el debate sobre la Revolución en los Asuntos Militares. Madrid: IUGM.

Coutau-Bégaire, Hervé. 2003. Traité de Stratégie. Paris: Económica.

Creveld, Martin V. 2000. "Through a Glass, Darkly: Some Reflections on the Future of Warfare". NetworkCentric Warfare Review 4 (3): 25-44.

Creveld, Martin V. 1991. The Transformation of War. Nueva York: The Free Press.

Denmark, Abraham y Mulvenon, James. 2010. Contested Commons: the Future of American Military Power in a Multipolar World. Washington DC: CNAS.

Department of Defense. 1993. Report on the Bottom-Up Review. Washington DC: GPO.

Department of Defense. 2001. Quadrennial Defense Review. Washington DC: GPO.

Department of Defense. 2003. Transformation Planning Guidance, Washington DC: GPO.

Department of Defense. 2005. National Defense Strategy. Washigton DC: GPO.

Department of Defense. 2010. Quadrennial Defense Review. Washington DC: GPO.

Department of Defense. 2011. Military and Security Developments Involving the People's Republic of China 2011. Washington DC: GPO.

Department of Defense. 2012. Sustaining U.S. Global Leadership: Priorities for $21^{\text {st }}$ Century Defense. Washington DC: GPO.

Eland, Iván. 2001. Putting "Defense" Back into U.S. Defense Policy: Rethinking U.S. Security in the Post-Cold War World. Westport: Praeger.

Flanagan, Stephen y Marti, Michael (eds.). 2003. The People's Liberation Army and China in Transition. Washington DC: National Defense University Press.

Friedman, George y Meredith. 1998. The Future of War: Power, Technology and American World Dominance in the Twenty-First Century. Nueva York: St. Martin's Griffin.

Government Accountability Office. 2010. Hybrid warfare. Washington DC: GAO.

Gvosdev, Nikolas y Reveron, Derek. 2010. "Waging War, Building States". Policy Review 163: 29-42.

Hays, Peter; Vallance, Brenda y Van Tassell, Allan (eds.). 1997 [1967]. American Defense Policy. Baltimore: John's Hopkins University Press.

Henrotin, Joseph. 2008. La technologie militaire en question. Le cas Américain. París: Económica.

Hoffman, Frank. 2007. Conflict in the 21 ${ }^{15 t}$ Century: the Rise of Hybrid Wars. Arlington: Potomac Institute for Policy Studies.

Kagan, Frederick. 2006. Finding the Target: The Transformation of American Military Policy. Nueva York: Encounter Books.

Kilcullen, David. 2009. The Accidental Guerrilla. Fighting Small Wars in the Midst of a Big One. Londres: Oxford University Press. 
Liang, Quiao y Xiangsui, Wang. 2004. La guerre hors limites. Paris: Rivages.

Lippitz, Michael y White, John (eds.). 2001. Keeping the Edge: Managing Defense for the Future. Cambridge: MIT Press.

Luttwak, Edward. 1987. Strategy: the Logic of War and Peace. Cambridge: Harvard University Press.

Ministry of National Defense. 2011. China's National Defense in 2008. Beijing: Information Office of the State Council of the People's Republic of China.

Moskos, Charles; Williams, James y Segal, Don. 2000. The Post-Modern Military: Armed Forces after the Cold War. Nueva York: Oxford University Press.

Owens, William. 1995. "The Emerging System-of-Systems". Proceedings 121 (1105): 35-39.

Peters, Ralph. 1995. "The Culture of Future Conflict". Parameters 25 (4): 18-27.

Rumsfeld, Donald. 2002. "Transforming the Military". Foreign Affairs 81 (3): 20-32.

Schneider, Barry y Grinter, Lawrence (eds.). 1998. Battlefield of the Future: $21^{\text {st }}$ Century Warfare Issues. Maxwell: AUP.

Snider, Don. 1993. Strategy, Forces and Budgets: Dominant Influences in Executive Decision Making, Post-Cold War 1989-91. Carlisle Barracks: SSI.

Sullivan, Gordon y Dubik, James. 1995. War in the Information Age. Carlisle Barracks: SSI.

Tomes, Robert. 2012. "Defense Strategy and Military Planning for an Era of Persistent Conflict". Small Wars Journal s.n. [http://smallwarsjournal.com/printpdf/12190]

Weigley, Russell. 1977. The American Way of War. Bloomington: Indiana University Press.

Guillem Colom Piella es profesor de Ciencia Política y de la Administración en la Universidad Pablo de Olavide y de Relaciones Internacionales en la Universidad Pontificia de Comillas (España). Es Doctor en Paz y Seguridad Internacional; Master en Derecho Internacional Público y Relaciones Internacionales y Licenciado en Sociología y en Ciencias Políticas. Sus líneas de investigación se centran en los asuntos estratégicos y políticas de seguridad y defensa, y entre sus trabajos destaca el libro Entre Ares y Atenea: el debate sobre la Revolución en los Asuntos Militares (IUGM, 2008). E-mail: gcolpie@upcomillas.es 